\title{
Determinantes de la Competitividad en el Sector Primario
}

\author{
Determinants of Competitiveness in the Primary Sector
}

\author{
Andrés Vergara-Narváez \\ Corporación Universitaria del Caribe (CECAR) - \\ Colombia \\ ORCID iD: https://orcid.org/0000-0003-3391-9235 \\ andres.vergaran@cecar.edu.co
}

Fecha de recepción: 03/01/2021

Fecha de evaluación: 27/01/2021

Fecha de aceptación: 22/03/2021

Cómo citar: Vergara-Narváez, A. (2021). Determinantes de la competitividad en el sector primario. Revista Cientifica Anfibios, 4(1). https://doi.org/10.37979/afb.2021v4n1.83

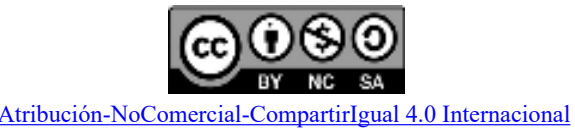

\section{Resumen}

Con el aumento exponencial de la población a nivel mundial y su respectiva demanda de alimentos, se requieren investigaciones que aborden los determinantes de competitividad del sector primario que permitan profundizar el conocimiento de la competitividad en este sector para que de esta manera se afronten mejor los restos y desafíos vinculados para la mejoras en la productividad. EN ese marco se plantea una revisión de la literatura que analice precisamente cuales son los determinantes de la competitividad en este sector. Adicionalmente se propone un análisis desde la óptica de las tipologías estratégicas con la finalidad de darle un valor agregado a esta revisión. Los resultados demuestran que el conocimiento acumulado, la eficiencia, el desarrollo tecnológico y la flexibilidad constituyen algunos elementos generadores de ventajas competitividad para el sector primario.

\section{Palabras Clave}

Sector agrícola; competitividad; escuelas de pensamiento competitivo; factores claves

\begin{abstract}
With the exponential increase in the world population and its respective demand for food, research is needed to address the determinants of competitiveness in the primary sector, which will allow for a deeper understanding of competitiveness in this sector so that the challenges and challenges related to productivity improvements can be better addressed. Within this framework, a literature review is proposed to analyze precisely what are the determinants of competitiveness in this sector. Additionally, an analysis from the perspective of strategic typologies is proposed in order to give added value to this review. The results show that accumulated knowledge, efficiency, technological development and flexibility are some of the elements that generate competitive advantages for the primary sector.
\end{abstract}

\section{Keywords}

Agricultural sector; competitiveness; competitive schools of thought; key factors 


\section{Introducción}

De acuerdo con las Naciones Unidas se estima que para el año 2050 la población mundial alcanzara alrededor de 9.700 millones de habitantes de las cuales el 2/3 habitaran en zonas urbanas, lo cual incrementara la demanda de alimentos (ONU, 2017). En ese sentido, existe la necesidad de aumentar la productividad agrícola de manera sostenible para atender esta creciente demanda. De manera similar, un informe de la FAO expresa que el aumento de la demanda de alimentos intensifica la competencia por los recursos naturales, junto con la deforestación y la degradación del suelo, haciendo que el futuro las perspectivas alimentarias a nivel mundial sean poco optimistas (FAO, 2017). No obstante, no hay que esperar a este escenario futuro para que salten las alarmas puesto que en la actualidad existen 800 millones de personas en el mundo pasando hambre de acuerdo con ese mismo informe y al considerar que la población mundial se estima en alrededor de 7 mil millones en 2018, este hecho es preocupante.

Es por esto que en 2016 fueron planteados los 17 Objetivos de Desarrollo Sostenible (ODS) por parte de las Naciones Unidas, dando inicio a una cuenta regresiva que finaliza el 2030 con el objetivo de cumplir 169 metas, entre las cuales muchas se asocian fundamentalmente con al desarrollo productivo del sector primario. Específicamente, el ODS 2 está centrado directamente en la alimentación buscando la erradicación el hambre, lograr la seguridad alimentaria acompaña de una mejor nutrición, y promover la explotación de recursos primarios sostenible, adicionalmente, el desarrollo del sector primario también se vincula con el ODS1 de mitigar la pobreza mundial, así como el ODS 6 relacionado con el uso del agua, una relación hacia el consumo y producción sostenible el ODS 12 con y el 15 respecto a la uso de la tierra y los ecosistemas, entre otros vínculos (FAO \& OCDE, 2016).

Este contexto hace un llamado tanto en lo académico como en lo práctico para estudiar los determinantes de la competitividad del sector primario y como mejorarlos con la finalidad de allanar el camino para el cumplimiento de estos ODS, en ese sentido, se plantea una revisión de la literatura que analice publicaciones científicas que permitan encontrar esos determinantes. Adi- cionalmente, estas publicaciones serán desarrolladas a partir de una óptica específica asociadas con tres tipologías estratégicas de competitividad, siendo estas; Miles \& Snow, (1978), Porter, (2000) y Miller (1987) que permita examinar estos determinantes a través de un prisma epistemológico que los contextualice de mejor manera. En ese sentido, las contribuciones planteadas se asocian con profundizar el estudio de los determinantes del sector primario haciendo especial énfasis en el sector agrícola, sin embargo, los hallazgos planteados pueden ser trasladados al sector ganadero, pesquero, minero y explotación forestal.

Para el cumplimiento de estos objetivos se plantea un apartado teórico en donde se plasman las principales visiones de las tres tipologías anteriormente mencionadas, seguidamente se mencionan los aspectos metodológicos que posibilitan encontrar publicaciones asociadas con los determinantes de la competitividad del sector primario. Posteriormente en los resultados se examinan las publicaciones científicas asociándolas con una de las tipologías propuestas con la finalidad de profundizar los análisis planteados. Finalmente se plantean las conclusiones y discusiones.

\section{Referentes Teóricos}

El concepto de competitividad ha tenido grandes evoluciones a lo largo de su historia, especialmente, en la literatura especializada debido a que cada sector económico define la competencia de acuerdo con su propia naturaleza, es por esto que se proponen diferentes visiones de la competitividad con énfasis en el sector primario.

Para Teece et al., (1997) competitividad se asocia con la medida en que un país produce bienes y servicios que satisfacen las necesidades del mercado en un mundo con clientes abiertos, al tiempo que expande su PIB y su PIB per cápita al menos tan rápido como sus socios comerciales. Por otra parte, Rojas \& Sepúlveda, (1999) expresan que esta competitividad implica la capacidad del sector para poner sus productos en el mercado en condiciones de competencia justa, lo que se traduce en el bienestar de las personas. En complemento Muller, (1995) sostiene que la competitividad es la habilidad de una organización para alcanzar, mantener e incrementar la participación de manera rentable para permitir su crecimiento. 
Adicionalmente, la competitividad se vincula con la habilidad de la organización para incrementar las ventas de productos y servicios y mantener sus cuotas de mercado sin renunciar a sus ganancias (Hernández, 2001)por una parte, los rasgos estilizados de la competitividad sistémica; por otra, las opciones de política pública de las pequeñas y medianas empresas (PYME.

Bajo este contexto y al examinar el concepto de competitividad del sector primario se expone la conceptualización propuesta por el Instituto Interamericano de Cooperación para la Agricultura (IICA), el cual define la competitividad como un concepto comparativo de posicionamiento espacial basado en las capacidades dinámicas de la cadena alimentaria agrícola para mantener, expandir y aumentar continuamente su participación de mercado. Esta habilidad obedece a muchos componentes a nivel macro, meso y micro. A nivel macro, se vincula con elementos relacionados con el país y sus vínculos comerciales con otras naciones buscando los intercambios adecuados es decir, adquirir lo que escasea y vender los viene abundantes; en un nivel meso económico, los componentes espaciales son relevantes como distancia, infraestructura de apoyo a la producción, base de recursos naturales e infraestructura social debido a que muchos costes intermedios pertenecen a este nivel. En el nivel micro, se destacan las organizaciones y sus características especifica como el precio y la calidad, (Romero \& Sepúlveda, 1999).

En este marco se reconoce el nuevo papel de la agricultura y el sector primario como catalizadores del crecimiento económico, mejorando la equidad, estabilizando la seguridad alimentaria, reduciendo la pobreza y el hambre además promoviendo el crecimiento económico y la sostenibilidad del medio ambiente (Byerlee et al., 2009). Por lo tanto, mediante el sector agropecuario, se puede contribuir al incremento del PIB y PIB per cápita del país, y de esa forma llegar a ser una nación competitiva (Teece et al., 1997)

Por otra parte, cabe resaltar que las características inherentes del sector primario así como su proceso productivo obstaculizan la generación de competitividad y diferenciación, debido a que la producción alberga bienes con poca diferenciación y estructuras de producción heterogéneas además de ser estacionales y que dependen de la geografía, del clima y de ciertas condiciones agroecológicas específicas que inciden en los niveles de producción. Adicionalmente la producción es inflexible, es decir, existen problemáticas para cambiar el uso y combinación de los factores productivos una vez iniciada la producción, mencionado que en muchos casos los factores no se usan en otros procesos; además, que la demanda de estos productos es principalmente para satisfacer las necesidades básicas, por lo cual la elasticidad es baja en el corto plazo (Bejarano, 1995).

Una vez mencionadas las principales características a nivel competitivo del sector primario se anuncian las 3 tipologías estratégicas que permitirán analizar los resultados de las publicaciones científicas.

Para Miles \& Snow, (1978) el éxito de una organización, independientemente de su sector, esta fuertemente vinculado con su habilidad para tomar decisiones y acciones que lleven al cumplimiento de sus objetivos, en ese sentido, la estrategia para stos autores radica en coordinar los recursos de la organización para el cumplimiento de metas. Sin embargo, esta estrategia depende en gran medida de la posición de la organización en ese momento clasificándose en cuatro tipos; exploradoras, defensoras, mixtas y reactivas. Las organizaciones exploradoras tienen por objetivo explorar y capitalizar nuevas oportunidades de mercado buscando la introducción de nuevos productos, servicios o procesos que dejen atrás a la competencia.

Por otra parte, las empresas defensoras son aquellas que no buscan incursionar en nuevos mercados si no proteger y asegurar su posición con decisiones conservadoras. No obstante, también existen organizaciones mixtas que puede que en algunos momentos sean exploradores buscando nuevas oportunidades de mercado mientras que en otro momento se vuelvan defensivas tratando se de asegurar su respectiva posición, sin embargo, puede que en algunas áreas comerciales la empresa sea exploradora y en otras sea defensora configurando una estrategia mixta. Por su parte, las empresas reactivas no responden ante cambios ni patrones del mercado, ni siquiera intentan mantener su posición si no simplemente están a la deriva dentro de su mercado.

Para Porter (2015) las estrategias empresariales vienen dadas por cinco fuerzas del mercado que influyen sobre la organización. La primera 
de estas fuerzas es el poder de negociación con el cliente en el cual los consumidores con un numero de exigencias creciente y con la posibilidad de comprar aquel producto y servicio que mejor satisfaga sus necesidades hace que las organizaciones este muy pendientes de este ante todo ofreciéndole calidad a precios adecuados. Por otra parte, la segunda fuerza obedece a los proveedores y se asocia con la capacidad de la organización para negociar las materias primas o los elementos necesarios para la producción, evidentemente se requiere una buena relación con proveedores para asegurar movimientos óptimos en la producción.

La tercera fuerza se asocia con la amenaza de nuevos competidores en donde le entrada de organizaciones con productos y servicios similares a los ofrecidos en el mercado es bastante latente, para contrarrestar esto la organización debe establecer barreras para que no ingresen nuevos competidores en el mercado. La cuarta fuerza también hace referencia a una amenaza pero esta de vez productos o servicios que sustituyan a los ya propuestos. Por ultimo la rivalidad entre los competidores el cual se deriva de todos los anteriores y coloca a la organización en una posición en la que debe atender muchos frentes, sin embargo, al considerar todos estos elementos es posible construir una estrategia que permita afrontar cada uno de estos componentes.

Por otra parte, Miller (1987) establece que las estrategias deben estar enfocadas hacia ciertas particularidades del mercado, en ese sentido, son cuatro las estrategias planteadas por el autor. La primera asociada con la innovación en donde la organización busca generar y alcanzar nuevas oportunidades de mercado por medio de la introducción de productos servicios o procesos. La segunda busca diferenciar el producto o servicio ofrecido por medio del marketing que represente un valor mayor para los consumidores que los ofrecidos por la competencia. La tercera estrategia se asocia con la amplitud en el sentido de enfocar segmentos de mercados específicos que posibiliten cierta estabilidad para la organización. La ultima estrategia se asocia con el control de costes en donde la organización se empeña en tener una producción alta de forma que el coste sea mínimo para maximizar sus ingresos.

En síntesis, se plantearon las principales características a nivel competitivo del sector pri- mario además de examinar unas tipologías estratégicas que permitan hacer una revisión de la literatura mas robusto.

\section{Metodología}

Tomando en consideración un enfoque cualitativo se pretende establecer los principales determinantes de la competitividad en el sector primario, para estos fines, se revisaron las investigaciones relevantes publicadas en revistas científicas de alto impacto sobre el tema, con el propósito de integrar y sintetizar el conocimiento generado, identificar áreas con mayor consenso y controversia, y explorar brechas y posibles direcciones de investigación.

Esta revisión de la literatura se planteo en dos pasos (Pujol-Cols \& Dabos, 2018). En el primer paso se realizó una búsqueda en plataformas de investigación científica como Scopus, Wos, Sicelo y Publindex con palabras claves como "competitividad" "Sector primario" "agroindustria". En el segundo paso se plantearon filtros para descartar registros que no esten directamente relacionados con el tema en investigación así como aquellas publicaciones que no demostraran sus resultados con datos empíricos. A partir de estos dos pasos se analizaron las publicaciones y se plantearon los resultados.

\section{Resultados}

La literatura de competitividad ha realizado estudios enfocado específicamente en el sector primario destacan una serie de factores y es necesario tener en cuenta que estos factores son diferentes a otros sectores económicos.

Marin Carrillo et al., (2004) analizan las fuerzas competitivas del sector agrícola de Almería, mencionando que los principales recursos en estudio obedecen al el clima, los recursos hídricos, el suelo, y que, considerando la riqueza agrícola y la capacidad para desarrollar nuevos productos, así como el conocimiento acumulado, la eficiencia, el desarrollo tecnológico y la flexibilidad se ha producido una ventaja competitiva con respecto a otros campos agrícolas en el mundo.

No obstante, este modelo de productividad Aún está restringido por factores ambientales, influenciado por competidores directos, distribuidores e 
incluso consumidores, quienes pueden cambiarlo; para dinamizarlo se han implementado estrategias comerciales. Adicionalmente, se planteó un análisis de la competitividad del sector tomando referenciando el modelo de las fuerzas competitivas de Porter estableciendo que el sistema productivo en estudio se constituye como uno de los modelos más exitosos a nivel nacional e internacional. En la actualidad, el sistema enfrenta nuevos desafíos, por lo que es necesario analizar las restricciones $\mathrm{u}$ obstáculos que dificultan la competitividad, especialmente el surgimiento de nuevos competidores y la comercialización de productos alternativos.

Díaz Cueva \& Mora Sanchez, (2016) plantearon una investigación en torno a los factores que se deben tener en cuenta para estudiar la competitividad de los productos asociados con el sector primario determinando que estos elementos Difíciles de identificar porque dependen de la cultura local y se enmarcan en la lógica de la agroindustria intensiva.

Adicionalmente, las organizaciones dedicadas al sector primario, en comparación con otro tipo de empresas, muestran características propias y diferenciadas, por lo que su investigación es particularmente necesaria. En ese sentido, los autores tomando como ejemplo a Ecuador, afirman que no existe una investigación o referencia para discutir los factores que afectan la competitividad de los productos agrícolas, esto también se puede ver en otros países de América Latina. Este trabajo es afín con la tipología de Miller en el sentido que debido a las características propias del sector primario se deben construir estrategias específicas que permitan a estas organizaciones explotar las mínimas diferencias que tengan y a partir de allí generar valor para su clientes.

Por su parte Saavedra García, (2012) Propone un método para determinar el nivel de competitividad de las pequeñas empresas, que considera estándares internos y externos mediante el análisis del nivel de competitividad por sector, por medio de una serie de antecedentes que permita la caracterización de la pyme en Latinoamérica, posteriormente Estudio en profundidad del concepto de competitividad, considerando indicadores externos e internos como tecnología, cultura, marketing, recursos humanos, innovación, logística, organización y calidad, todo ello basado en una revisión de teorías planteadas por diferentes autores.
Esta publicación concluye que Llegando a concluir que muy Si bien la mayoría de los autores proponen indicadores desde un ámbito interno, consideran que los indicadores externos son muy importantes porque no pueden aumentar la competitividad sin conocer el entorno, por lo que los autores propusieron el desarrollo del Map Bank of America para determinar los factores internos y la competitividad sistémica propuesta por la CEPAL.

Desde el punto de vista de la tipología estratégica el trabajo planteado por Saavedra García, (2012) se ubica dentro de las 5 fuerzas de Porter en el sentido en que esta autora entiende que las fuentes de competitividad de las organizaciones ubicadas en el sector primario se deben a la interacción de esta fuerzas determinando que solo con un análisis holístico de cada una de estas fuerzas ser posible comenzar a construir valor para los clientes.

Castaño Giraldo \& Cardona Gómez, (2014) a través de la revisión y análisis de documentos por expertos, se revelan los factores que inciden en la inestabilidad del sector agrícola conformando que Colombia es uno de los países con mayor potencial para expandir la tierra agrícola; sin embargo, la participación del sector en el PIB nacional ha venido disminuyendo en los últimos años. Mala infraestructura vial, desplazamiento y una serie de problemas relacionados con la legalización y la devolución de tierras han tropezado con tales dificultades.

Al igual que los autores mencionados, en el capítulo sobre la competitividad del sector agropecuario colombiano, confirmaron que el sector ha ido perdiendo vitalidad en los últimos años, aunque el sector es uno de los principales generadores de empleo a nivel nacional, especialmente en las zonas rurales. y esto también es fundamental para el suministro de alimentos y materias primas que se utilizan para producir el producto final. Como resultado, pudieron determinar la implementación de varios tratados de libre comercio negociados en los últimos años, los cuales desencadenaron una evaluación del desempeño del departamento y analizaron las principales debilidades relacionadas con el bajo nivel de capital humano, la escasez de tecnología y su aporte. al departamento Transfer. Todas estas dificultades deben resolverse de acuerdo con el principio de sostenibilidad, basado en los tres pilares de la 
economía, la sociedad y el medio ambiente (Consejo Privado de Competitividad, 2017).

\section{Conclusiones y Discusiones}

Ante el aumento exponencial de la población mundial y su respectiva creciente demanda de alimentos, existe la necesidad de estudiar en profundidad como mejorar la competitividad del sector primario que posibilite entender en mejor medida este sector y a partir de allí comenzar a mejorar su productividad. Ante este panorama se planteó una revisión de la literatura que por analizar las publicaciones científicas que trataran esta temática aportando con datos empíricos sus resultados.

Es así que los elementos que se identificaron como claves dentro de la teoría de competitividad del sector primario se asocian con tecnología, cultura, marketing, recursos humanos, innovación, logística, organización y calidad. En ese marco se realizaron algunos análisis desde las tipologías estratégicas de autores destacados dando una arista epistemológica que permitió profundizar el análisis.

Todo lo anterior permite plantear una discusión respecto a los elementos fundamentales para aumentar la competitividad en el sector primario, en ese sentido, las políticas publicas y los sectores privados deben fortalecer el aparato productor del sector primario de tal manera que se afiance su competitividad y comenzar a trabajar en post de atender la creciente demanda alimentaria y mejor el nivel económico de las familias que dependen de este sector.

\section{Referencias}

Byerlee, D., de Janvry, A., \& Sadoulet, E. (2009). Agriculture for Development: Toward a New Paradigm. Annual Review of Resource Economics, 1(1), 15-31. https://doi.org/10.1146/annurev.resource.050708.144239

Castaño Giraldo, N. E., \& Cardona Gómez, M. A. (2014). Factores determinantes en la inestabilidad del sector agrícola colombiano. En Contexto, 2, 91-107.

Díaz Cueva, J., \& Mora Sanchez, N. (2016). Análisis de los factores a tomar en cuenta para el estudio de la competitividad de los productos agrícolas. Revista Publicando, 3(8), 539-552.

FAO. (2017). El Futuro de la Alimentación. 1.

Fao, \& Ocde. (2016). OCDE-FAO Perspectivas Agrícolas 2016-2025. https://doi.org/10.1787/agr_outlook-2018-es

Hernández, R. (2001). Elementos de Competitividad Sistemica de las PYMEs del Istmo Centroamericano. In Naciones Unidas - CEPAL: Vol. MEX (Issue L.499).

Marin Carrillo, M. B., Marin Carrillo, G. M., \& Jimenez Castillo, D. J. (2004). Análisis de las fuerzas competitivas del sector agrícola de Almería. Boletín Económico de ICE, Información Comercial Española, 2798, 47-60.

Miles, R., \& Snow, C. (1978). Organizational Strategy, Structure, and Process. Mc Graw Hil.

Muller, G. (1995). El caleidoscopio de la competitividad. Revista De La Cepal, 56, 137-148.

ONU. (2017). Población | Naciones Unidas. Población.

Porter, M. (2015). Estratégia competitiva (6ta edicio). Editorial continental S.A.

Porter, Michael. (2000). Location, Competition, and Economic Development : Local Clusters in a Global. Harvard Business School, 14(1), 15-34. https://doi.org/10.1177/089124240001400105

Pujol-Cols, L. J., \& Dabos, G. E. (2018). Satisfacción laboral: una revisión de la literatura acerca de sus principales determinantes. Estudios Gerenciales, 34(146), 3-18. https://doi.org/10.18046/j.estger.2018.146.2809 
Rojas, P., \& Sepúlveda, S. (1999). El reto de la competitividad en la agricultura. 24.

Romero, S., \& Sepúlveda, S. (1999). Territorio, agricultura y competitividad. Cuadernos Técnicos, $10,21$.

Saavedra García, M. L. (2012). Una propuesta para la determinación de la competitividad en la pyme latinoamericana. Pensamiento \& Gestión, 33(1), 93-124.

Teece, D. J., Pisano, G., \& Shuen, A. (1997). Dynamic capabilities and strategic management. Strategic Management Journal, 18(7), 509-533. https://doi.org/10.1002/(SICI)10970266(199708)18:7<509::AID-SMJ882>3.0.CO;2-Z 